\title{
A PROOF OF BOUNDEDNESS OF THE CARLESON OPERATOR
}

\author{
Michael Lacey and Christoph Thiele
}

ABSTRACT. We give a simplified proof that the Carleson operator is of weak type $(2,2)$. This estimate is the main ingredient in the proof of Carleson's theorem on almost everywhere convergence of Fourier series of functions in $L^{2}([0,1])$.

\section{Introduction}

We define the Carleson operator $\mathcal{C}$ acting on a Schwartz function $f$ on $\mathbb{R}$ by

$$
\mathcal{C} f(x):=\sup _{N}\left|\int_{-\infty}^{N} \widehat{f}(\xi) e^{2 \pi i \xi x} d \xi\right|,
$$

where the Fourier transform $\widehat{f}$ is defined by

$$
\widehat{f}(\xi):=\int f(x) e^{-2 \pi i \xi x} d x .
$$

We give a simplified proof of the well known theorem [1], [2]:

Theorem 1.1. The Carleson operator $\mathcal{C}$ is of weak type $(2,2)$, i.e.,

$$
\|\mathcal{C} f\|_{L^{2, \infty}} \leq C\|f\|_{2}
$$

with a constant $C$ not depending on $f$.

This theorem is the key ingredient in the proof of Carleson's celebrated theorem, which asserts that the Fourier series of a function in $L^{2}([0,1])$ converges pointwise almost everywhere.

We became interested in Carleson's theorem while studying the bilinear Hilbert transform [3], [4], [5], [6]. As it turns out, these two subjects are closely related. The purpose of the current article is to exhibit these connections by giving a proof of Carleson's theorem in the spirit of [3], [4], [5], [6]. In particular, the key Proposition 3.2 below is essentially taken from these papers.

While L. Carleson [1] uses a decomposition of the function $f$ and C. Fefferman [2] features a decomposition of the Carleson operator guided by $N$ (the function which picks the worst $N$ for each $x$ in the Carleson operator), we emphasize a symmetry between $f$ and $N$, as expressed by the duality of Propositions 3.1

Received September 23, 1999.

The authors were supported by NSF grants DMS 9706884 and DMS 9970469 respectively, their collaboration was supported by NATO grant CRG 960113. 
and 3.2. This symmetry is more perfect in the case of the bilinear Hilbert transform, where instead of $f$ and $N$ one has three Schwartz functions $f_{1}, f_{2}$, and $f_{3}$, and a variant of Proposition 3.2 is applied to all three of them.

In Section 2 we introduce most of the notation used in this paper, and we do a discretization of the Carleson operator. In Section 3 we prove boundedness of the discretized Carleson operator by taking for granted Propositions 3.1 and 3.2 and some technical inequality (8) from standard singular integral theory. These remaining items are proved in Sections 4,5 , and 6 .

The authors would like to thank the referee and John Garnett for their helpful comments on previous versions of the manuscript.

\section{Notation and preliminary reductions}

Define translation, modulation, and dilation operators by

$$
\begin{aligned}
T_{y} f(x) & =f(x-y), \\
M_{\eta} f(x) & =f(x) e^{2 \pi i \eta x}, \\
D_{\Lambda}^{p} f(x) & =\Lambda^{-\frac{1}{p}} f\left(\Lambda^{-1} x\right) .
\end{aligned}
$$

We write $|E|$ for the measure of a set $E \subset \mathbb{R}^{n}$. By an interval we mean a subset $[x, y)$ of $\mathbb{R}$ with $x<y$. We write $c(I)$ for the center of the interval $I$, and $\alpha I$ for the interval with the same center and $\alpha$ times the length of $I$, where $\alpha>0$. We write $1_{I}$ for the characteristic function of $I$ and define weight functions

$$
w(x):=(1+|x|)^{-\nu}, \quad w_{I}(x):=T_{c(I)} D_{|I|}^{1} w,
$$

where the letter $\nu$ as in the rest of the paper is used for a large integer whose exact value is not important and may be different at different places of the argument.

Let $\phi$ be a Schwartz function such that $\widehat{\phi}$ is real, non-negative, supported in $[-0.1,0.1]$, and equal to 1 on $[-0.09,0.09]$. For each rectangle $P=I_{P} \times \omega_{P}$ of area 1 in the (phase-) plane define

$$
\phi_{1 P}:=M_{c\left(\omega_{1 P}\right)} T_{c\left(I_{P}\right)} D_{\left|I_{P}\right|}^{2} \phi,
$$

where we have written $\omega_{1 P}$ for the lower half $\omega_{P} \cap\left(-\infty, c\left(\omega_{P}\right)\right)$ of $\omega_{P}$. Similarly we write $\omega_{2 P}$ for the upper half $\omega_{P} \backslash \omega_{1 P}$. Observe that $\widehat{\phi}_{1 P}$ is supported in $\frac{1}{2} \omega_{1 P}$ and we have

$$
\left|\phi_{1 P}(x)\right| \leq C\left|I_{P}\right|^{\frac{1}{2}} w_{P}(x),
$$

where $w_{P}:=w_{I_{P}}$ and $C$ denotes as in the rest of the paper a large number whose value depends only on the choice of $\phi$ and $\nu$ and may be different at different places of the argument.

A dyadic interval is of the form $\left[n 2^{k},(n+1) 2^{k}\right)$ with integers $n$ and $k$. Let $\overline{\mathbf{P}}$ denote the set of rectangles $I \times \omega$ with $I, \omega$ dyadic and $|I||\omega|=1$. Define

$$
A_{\xi} f:=\sum_{P \in \overline{\mathbf{P}}} 1_{\omega_{2 P}}(\xi)\left\langle f, \phi_{1 P}\right\rangle \phi_{1 P} .
$$


Observe that for every integer $k$ we have

$$
A_{\xi} f=D_{2^{-k}}^{2} A_{2^{-k} \xi} D_{2^{k}}^{2} f .
$$

Averaging over translates and dilates of the involved dyadic structures we define

$$
\Pi_{\xi} f:=\lim _{n \rightarrow \infty} \frac{1}{\left|K_{n}\right|} \int_{K_{n} \times[0,1]} M_{-\eta} T_{-y} D_{2^{-\kappa}}^{2} A_{2^{-\kappa}(\eta+\xi)} D_{2^{\kappa}}^{2} T_{y} M_{\eta} f d y d \eta d \kappa,
$$

where $K_{n}$ is any increasing sequence of rectangles $I_{n} \times \omega_{n}$ filling out $\mathbb{R}^{2}$. To see the pointwise convergence of the last expression, consider separately those rectangles $P \in \overline{\mathbf{P}}$ with $\left|I_{P}\right|$ fixed, then the integrand becomes periodic in $y$ and $\eta$, and observe that for very large and very small values of $\left|I_{P}\right|$ the integrand becomes small. It is easy to verify that $\Pi_{\xi}$ extends to a bounded operator on $L^{2}$, is nonzero and positive semidefinite, commutes with $T_{y}$ for all $y$ and with $D_{\lambda}^{2} M_{(\lambda-1) \xi}$ for all $\lambda>0$, and satisfies $\Pi_{\xi} f=0$ if $\widehat{f}$ is supported in $[\xi, \infty]$. This identifies $\Pi_{\xi}$ as

$$
\Pi_{\xi} f(x)=c_{\xi} \int_{-\infty}^{\xi} \widehat{f}(\eta) e^{2 \pi i x \eta} d \eta
$$

for some constant $c_{\xi} \neq 0$. By conjugating with $M_{\xi^{\prime}}$ one observes that $c=c_{\xi}$ does not depend on $\xi$. Hence the Carleson operator is equal to

$$
\mathcal{C} f(x)=c^{-1} \sup _{\xi}\left|\Pi_{\xi} f(x)\right| \text {. }
$$

We will prove that

$$
\left\|\sup _{\xi}\left|A_{\xi} f\right|\right\|_{L^{2, \infty}} \leq C\|f\|_{2} .
$$

By averaging this implies

$$
\begin{aligned}
& \left\|\sup _{\xi}\left|\Pi_{\xi} f(x)\right|\right\|_{2, \infty} \\
& \quad \leq \limsup _{n} \frac{1}{\left|K_{n}\right|} \int\left\|\sup _{\xi}\left|A_{\xi} D_{2^{\kappa}} T_{y} M_{\eta} f\right|\right\|_{2, \infty} d y d \eta d \kappa \leq C\|f\|_{2},
\end{aligned}
$$

which is enough to conclude Theorem 1.1.

By duality and the triangle inequality estimate (2) follows from

$$
\sum_{P \in \mathbf{P}}\left|\left\langle f, \phi_{1 P}\right\rangle\left\langle\phi_{1 P}\left(1_{\omega_{2 P}} \circ N\right), 1_{E}\right\rangle\right| \leq C\|f\|_{2}|E|^{\frac{1}{2}}
$$

for all Schwartz functions $f$, measurable functions $N$, measurable sets $E$, and finite subsets $\mathbf{P}$ of $\overline{\mathbf{P}}$. Since this estimate is homogeneous in $f$ and invariant under appropriate simultaneous dilations of $f, N, E$, and $\mathbf{P}$, it suffices to prove the estimate for $\|f\|_{2}=1$ and $|E| \leq 1$. With $E$ fixed for the rest of the paper, we write

$$
E_{P}:=E \cap\left\{x: N(x) \in \omega_{P}\right\}, \quad E_{2 P}:=E \cap\left\{x: N(x) \in \omega_{2 P}\right\} .
$$




\section{The main argument}

A rectangle $P=I_{P} \times \omega_{P}$ of $\overline{\mathbf{P}}$ will be called a tile. Each tile has area 1 and is the union of two semitiles $P_{1}=I_{P} \times \omega_{1 P}$ and $P_{2}=I_{P} \times \omega_{2 P}$. Observe that dyadic intervals such as $I_{P}, \omega_{P}, \omega_{j P}$ have the property that any two of them are either disjoint or one is contained in the other. Moreover, if $\omega_{1 P}$ is strictly contained in a dyadic interval, then $\omega_{2 P}$ is strictly contained in the same interval and vice versa. We will use these geometric properties without referring to them. We define a partial ordering on the set of tiles by $P<P^{\prime}$ if $I_{P} \subset I_{P^{\prime}}$ and $\omega_{P^{\prime}} \subset \omega_{P}$.

A set $T$ of tiles is called a tree, if there is a tile $P_{T}=I_{T} \times \omega_{T}$, the top of the tree, such that $P<P_{T}$ for all $P \in T$. Observe that we do not require the top to be an element of the tree. A tree is called $j$-tree if $\omega_{j P_{T}} \subset \omega_{j P}$ for all $P \in T$.

For the following definitions and propostitions recall that we are given data $E$, $f$, and $N$ with $|E| \leq 1$ and $\|f\|_{2}=1$. For a finite subset $\mathbf{P} \subset \overline{\mathbf{P}}$, define

$$
\begin{gathered}
\operatorname{mass}(\mathbf{P}):=\sup _{P \in \mathbf{P}} \sup _{P^{\prime} \in \overline{\mathbf{P}}: P<P^{\prime}} \int_{E_{P^{\prime}}} w_{P^{\prime}}(x) d x, \\
\operatorname{energy}(\mathbf{P}):=\sup _{T}\left(\left|I_{T}\right|^{-1} \sum_{P \in T}\left|\left\langle f, \phi_{1 P}\right\rangle\right|^{2}\right)^{\frac{1}{2}},
\end{gathered}
$$

where the sup in the definition of energy is taken over all 2-trees $T \subset \mathbf{P}$.

Proposition 3.1. Let $\mathbf{P}$ be a finite set of tiles, then $\mathbf{P}$ can be decomposed as the union of sets $\mathbf{P}_{\text {light }}$ and $\mathbf{P}_{\text {heavy }}$ with

$$
\operatorname{mass}\left(\mathbf{P}_{\text {light }}\right) \leq 2^{-1} \operatorname{mass}(\mathbf{P}),
$$

and $\mathbf{P}_{\text {heavy }}$ is the union of a set $\mathbf{T}$ of trees such that

$$
\sum_{T \in \mathbf{T}}\left|I_{T}\right| \leq C \operatorname{mass}(\mathbf{P})^{-1} .
$$

Proposition 3.2. Let $\mathbf{P}$ be a finite set of tiles, then $\mathbf{P}$ can be decomposed as the union of $\mathbf{P}_{\text {low }}$ and $\mathbf{P}_{\text {high }}$ with

$$
\operatorname{energy}\left(\mathbf{P}_{\text {low }}\right) \leq 2^{-1} \operatorname{energy}(\mathbf{P}),
$$

and $\mathbf{P}_{\text {high }}$ is the union of a set $\mathbf{T}$ of trees such that

$$
\sum_{T \in \mathbf{T}}\left|I_{T}\right| \leq C \operatorname{energy}(\mathbf{P})^{-2} .
$$

We prove these propositions in Sections 4 and 5. Given a finite collection $\mathbf{P}$ of tiles, we use Propositions 3.1 and 3.2 to obtain a decomposition of $\mathbf{P}$ into sets $\mathbf{P}_{n}$, where $n$ runs through some finite set of integers, such that for each $n$ we have

$$
\operatorname{mass}\left(\mathbf{P}_{n}\right) \leq 2^{2 n}, \quad \operatorname{energy}\left(\mathbf{P}_{n}\right) \leq 2^{n}
$$

and $\mathbf{P}_{n}$ is the union of a set $\mathbf{T}_{n}$ of trees with

$$
\sum_{T \in \mathbf{T}_{n}}\left|I_{T}\right| \leq C 2^{-2 n} .
$$


Namely, initially $\mathbf{P}$ satisfies estimates as in (6) for some large $n$. If the mass of $\mathbf{P}$ is greater than $2^{2(n-1)}$, we split it into $\mathbf{P}_{\text {light }}$ and $\mathbf{P}_{\text {heavy }}$, replace $\mathbf{P}$ by $\mathbf{P}_{\text {light }}$, and add $\mathbf{P}_{\text {heavy }}$ to $\mathbf{P}_{n}$. Then, if the energy of $\mathbf{P}$ is greater than $2^{n-1}$, we split $\mathbf{P}$ into $\mathbf{P}_{\text {high }}$ and $\mathbf{P}_{\text {low }}$, replace $\mathbf{P}$ by $\mathbf{P}_{\text {low }}$, and add $\mathbf{P}_{\text {high }}$ to $\mathbf{P}_{n}$. Then $\mathbf{P}$ satisfies (6) with $n$ replaced by $n-1$ and we iterate.

In Section 6 we prove for each tree $T$ the inequality

$$
\sum_{P \in T}\left|\left\langle f, \phi_{1 P}\right\rangle\left\langle\phi_{1 P}, 1_{E_{2 P}}\right\rangle\right| \leq C \operatorname{energy}(T) \operatorname{mass}(T)\left|I_{T}\right| .
$$

Hence, with the observation that the mass of any collection of tiles is bounded by a universal constant, we have the estimate

$$
\sum_{T \in \mathbf{T}_{n}} \sum_{P \in T}\left|\left\langle f, \phi_{1 P}\right\rangle\left\langle\phi_{1 P}, 1_{E_{2 P}}\right\rangle\right| \leq C 2^{n} \min \left(C, 2^{2 n}\right) 2^{-2 n} .
$$

This is summable over $n \in \mathbb{Z}$, which proves (3) and therefore Theorem 1.1.

\section{Proof of Proposition 3.1}

Let $\mu=\operatorname{mass}(\mathbf{P})$. Let $\mathbf{P}_{\text {heavy }}$ be the set of tiles $P \in \mathbf{P}$ with $\operatorname{mass}(\{P\})>$ $2^{-1} \mu$. To each such tile $P$ we may associate a tile $P^{\prime}(P)$ with $P<P^{\prime}(P)$ and

$$
\int_{E_{P^{\prime}(P)}} w_{P^{\prime}(P)} d x>2^{-1} \mu \text {. }
$$

Then, let $\mathbf{P}^{\prime}$ be those elements in $\left\{P^{\prime}(P) \mid P \in \mathbf{P}_{\text {heavy }}\right\}$ which are maximal with respect to the partial order $<$ of tiles. It suffices to show that

$$
\sum_{P^{\prime} \in \mathbf{P}^{\prime}}\left|I_{P^{\prime}}\right|<C \mu^{-1}
$$

because the tiles $P \in \mathbf{P}_{\text {heavy }}$ can be collected into trees with tops in $\mathbf{P}^{\prime}$.

For $\kappa \in \mathbb{N}$ define $\mathbf{P}_{\kappa}$ to be the set of all $P \in \mathbf{P}^{\prime}$ with

$$
\left|E_{P} \cap 2^{\kappa} I_{P}\right| \geq c 2^{2 \kappa} \mu\left|I_{P}\right|
$$

for some constant $c$. If $c$ is small enough, then one can conclude from the mass estimate that each element $P$ of $\mathbf{P}^{\prime}$ is contained in one of the sets $\mathbf{P}_{\kappa}$. Hence it suffices to show for every $\kappa$

$$
\sum_{P \in \mathbf{P}_{\kappa}}\left|I_{P}\right| \leq C 2^{-\kappa} \mu^{-1} .
$$

Fix $\kappa$. For each $P \in \mathbf{P}_{\kappa}$ we have an enlarged rectangle $\left(2^{\kappa} I_{P}\right) \times \omega_{P}$. We select successively elements $P \in \mathbf{P}_{\kappa}$ with maximal $\left|I_{P}\right|$ whose enlarged rectangles are disjoint from the enlarged rectangles of all previously selected elements. When no further element can be selected, then each rectangle $P^{\prime} \in \mathbf{P}_{\kappa}$ can be associated to a selected rectangle $P$ such that $\left|I_{P^{\prime}}\right|<\left|I_{P}\right|$ and the enlarged rectangles of $P$ and $P^{\prime}$ intersect. Since the rectangles in $\mathbf{P}_{\kappa}$ are pairwise disjoint, we see that the intervals $I_{P^{\prime}}$ of the rectangles $P^{\prime}$ associated to a fixed selected $P$ are pairwise disjoint and contained in $2^{\kappa+2} I_{P}$. Hence

$$
\sum_{P \in \mathbf{P}_{\kappa}}\left|I_{P}\right| \leq C 2^{\kappa} \sum_{P \text { selected }}\left|I_{P}\right| \leq C 2^{-\kappa} \mu^{-1} \sum_{P \text { selected }}\left|E_{P} \cap 2^{\kappa} I_{P}\right| .
$$


This is bounded by $C 2^{-\kappa} \mu^{-1}$ because the enlarged rectangles of the selected elements $P$ are pairwise disjoint and therefore the subsets $E_{P} \cap 2^{\kappa} I_{P}$ of $E$ are pairwise disjoint. This finishes the proof of Proposition 3.1.

\section{Proof of Proposition 3.2}

Let $\varepsilon=\operatorname{energy}(\mathbf{P})$. For a 2 -tree $T$, let

$$
\Delta(T)^{2}=\left|I_{T}\right|^{-1} \sum_{P \in T}\left|\left\langle f, \phi_{1 P}\right\rangle\right|^{2} .
$$

We inductively construct the collection $\mathbf{T}$ of trees whose union will be $\mathbf{P}_{\text {high }}$.

Pick a 2-tree $T \in \mathbf{P}$ such that (1) $\Delta(T) \geq 2^{-1} \varepsilon$ and $(2) c\left(\omega_{T}\right)$ is minimal among all 2-trees satisfying the first condition. Then let $T^{1}$ be the maximal (with respect to set inclusion) tree in $\mathbf{P}$ with top $I_{T} \times \omega_{T}$.

Add $T^{1}$ to $\mathbf{T}$, add $T$ to $\mathbf{T}_{2}$, which will be a collection of 2 -trees we will work with in the sequel because it has better disjointness properties than the collection $\mathbf{T}$. Remove each element of $T^{1}$ from $\mathbf{P}$. Then repeat the procedure above until there is no tree in $\mathbf{P}$ with $\Delta(T) \geq 2^{-1} \varepsilon$. Then we can define $\mathbf{P}$ to be $\mathbf{P}_{\text {light }}$.

Let $T, T^{\prime} \in \mathbf{T}_{2}$ and let $P \in T$ and $P^{\prime} \in T^{\prime}$. If $\omega_{P}$ is contained in $\omega_{1 P^{\prime}}$, then $I_{P^{\prime}} \cap I_{T}=\emptyset$. To see this, note that $c\left(\omega_{T}\right)$, which is contained in $\omega_{P}$, is less than $c\left(\omega_{T^{\prime}}\right) \in \omega_{2 P^{\prime}}$. Therefore $T$ was selected before $T^{\prime}$. But if $I_{P^{\prime}}$ and $I_{T}$ had nonempty intersection, then $P^{\prime}$ would qualify to be in the tree $T^{1}$ and would have been removed from $\mathbf{P}$ before $T^{\prime}$ was selected. This is impossible.

It remains to show that

$$
\varepsilon^{2} \sum_{T \in \mathbf{T}_{2}}\left|I_{T}\right| \leq C
$$

Letting $\overline{\mathbf{P}}$ be the union of the 2 -trees $T$ in $\mathbf{T}_{2}$, the left hand side is at most a constant times

$$
\sum_{P \in \overline{\mathbf{P}}}\left|\left\langle f, \phi_{1 P}\right\rangle\right|^{2} \leq\left\|\sum_{P \in \overline{\mathbf{P}}}\left\langle f, \phi_{1 P}\right\rangle \phi_{1 P}\right\|_{2} .
$$

Here we have used that the $L^{2}$-norm of $f$ is 1 .

Therefore, it is sufficient to prove

$$
\left\|\sum_{P \in \overline{\mathbf{P}}}\left\langle f, \phi_{1 P}\right\rangle \phi_{1 P}\right\|_{2}^{2} \leq C \varepsilon^{2} \sum_{T \in \mathbf{T}_{2}}\left|I_{T}\right| .
$$

We estimate the left hand side of (9) by

$$
\begin{aligned}
& \sum_{P, P^{\prime} \in \overline{\mathbf{P}}: \omega_{P}=\omega_{P^{\prime}}}\left|\left\langle f, \phi_{1 P}\right\rangle\left\langle\phi_{1 P}, \phi_{1 P^{\prime}}\right\rangle\left\langle\phi_{1 P^{\prime}}, f\right\rangle\right| \\
& +2 \sum_{P, P^{\prime} \in \overline{\mathbf{P}}: \omega_{P} \subset \omega_{1 P^{\prime}}}\left|\left\langle f, \phi_{1 P}\right\rangle\left\langle\phi_{1 P}, \phi_{1 P^{\prime}}\right\rangle\left\langle\phi_{1 P^{\prime}}, f\right\rangle\right| .
\end{aligned}
$$

Here we have used symmetry and the fact that $\left\langle f, \phi_{1 P}\right\rangle=0$ unless one of the intervals $\omega_{1 P}$ and $\omega_{1 P^{\prime}}$ is contained in the other.

Observe that for $\left|I_{P^{\prime}}\right| \leq\left|I_{P}\right|$ we have

$$
\left|\left\langle\phi_{1 P}, \phi_{1 P^{\prime}}\right\rangle\right| \leq C\left|I_{P}\right|^{\frac{1}{2}}\left|I_{P^{\prime}}\right|^{-\frac{1}{2}}\left\|w_{P} 1_{I_{P^{\prime}}}\right\|_{1} .
$$


We estimate the smaller one of $\left|\left\langle f, \phi_{1 P}\right\rangle\right|$ and $\left|\left\langle f, \phi_{1 P^{\prime}}\right\rangle\right|$ by the larger one and use symmetry to obtain for (10) the upper bound

$$
C \sum_{P \in \overline{\mathbf{P}}}\left|\left\langle f, \phi_{1 P}\right\rangle\right|^{2} \sum_{P^{\prime} \in \overline{\mathbf{P}}: \omega_{P}=\omega_{P^{\prime}}}\left\|w_{P} 1_{I_{P^{\prime}}}\right\|_{1} .
$$

The interior sum we can estimate by $\left|I_{P}\right|^{-1}\left\|w_{P}\right\|_{1} \leq C$, because the intervals $I_{P^{\prime}}$ with $\omega_{P^{\prime}}=\omega_{P}$ are pairwise disjoint. This proves the desired bound for (10).

The second summand (11) we estimate by

$$
\begin{aligned}
\sum_{P \in \overline{\mathbf{P}}}\left|\left\langle f, \phi_{1 P}\right\rangle\right| & \sum_{P^{\prime} \in \overline{\mathbf{P}}: \omega_{P} \subset \omega_{1 P^{\prime}}}\left|\left\langle\phi_{1 P}, \phi_{1 P^{\prime}}\right\rangle\left\langle\phi_{1 P^{\prime}}, f\right\rangle\right| \\
\leq & \sum_{T \in \mathbf{T}_{2}}\left(\sum_{P \in T}\left|\left\langle f, \phi_{1 P}\right\rangle\right|^{2}\right)^{\frac{1}{2}} H(T)^{\frac{1}{2}} \leq C \varepsilon \sum_{T \in \mathbf{T}_{2}}\left|I_{T}\right|^{\frac{1}{2}} H(T)^{\frac{1}{2}}
\end{aligned}
$$

where

$$
H(T):=\sum_{P \in T}\left(\sum_{P^{\prime} \in \overline{\mathbf{P}}: \omega_{P} \subset \omega_{1 P^{\prime}}}\left|\left\langle\phi_{1 P}, \phi_{1 P^{\prime}}\right\rangle\left\langle\phi_{1 P^{\prime}}, f\right\rangle\right|\right)^{2}
$$

It remains to show that $H(T) \leq C \varepsilon^{2}\left|I_{T}\right|$ for each tree $T \in \mathbf{T}_{2}$.

But,

$$
H(T) \leq C \varepsilon^{2} \sum_{P \in T}\left|I_{P}\right|\left(\sum_{P^{\prime} \in \overline{\mathbf{P}}: \omega_{P} \subset \omega_{1 P^{\prime}}}\left\|w_{P} 1_{I_{P^{\prime}}}\right\|_{1}\right)^{2},
$$

where we have used the upper energy estimate for each individual $P^{\prime}$ (which is a 2 -tree by itself), and the estimate on $\left\langle\phi_{1 P}, \phi_{1 P^{\prime}}\right\rangle$. Fix $P$, then the intervals $I_{P^{\prime}}$ with $\omega_{P} \subset \omega_{1 P^{\prime}}$ are pairwise disjoint and disjoint from $I_{T}$ by the above mentioned disjointness property. Hence we have

$$
\sum_{P^{\prime} \in \overline{\mathbf{P}}: \omega_{P} \subset \omega_{1 P^{\prime}}}\left\|w_{P} 1_{I_{P^{\prime}}}\right\|_{1} \leq C\left\|w_{P} 1_{I_{T}^{c}}\right\|_{1} .
$$

For each $x \in I_{T}$ there is at most one $P \in T$ of each scale with $x \in I_{P}$. Hence we have:

$$
\begin{aligned}
\sum_{P \in T}\left|I_{P}\right|\left\|w_{P} 1_{I_{T}^{c}}\right\|_{1}^{2} & \leq C \sum_{P \in T}\left|I_{P}\right|\left\|w_{P} 1_{I_{T}^{c}}\right\|_{1} \\
& \leq C \sum_{k \in \mathbb{N}}\left\|\left(1_{I_{T}} * D_{2^{-k}\left|I_{T}\right|}^{1} w\right) 1_{I_{T}^{c}}\right\|_{1} \leq C\left|I_{T}\right| .
\end{aligned}
$$

This gives the appropriate bound for $H(T)$ and thus finishes the proof of (9). 


\section{Proof of estimate (8)}

Let $\mathcal{J}$ be the collection of all maximal dyadic intervals such that $3 J$ does not contain any $I_{P}$ with $P \in T$. Then $\mathcal{J}$ is a partition of $\mathbb{R}$.

We estimate the left hand side of (8) as below, where the terms $\epsilon_{P}$ are phase factors of modulus 1 which make up for the absolute value signs in (8).

$$
\left\|\sum_{P \in T} \epsilon_{P}\left\langle f, \phi_{1 P}\right\rangle \phi_{1 P} 1_{E_{2 P}}\right\|_{1} \leq
$$

$$
\begin{aligned}
\sum_{J \in \mathcal{J}} & \sum_{P \in T:\left|I_{P}\right| \leq|J|}\left\|\left\langle f, \phi_{1 P}\right\rangle \phi_{1 P} 1_{E_{2 P}}\right\|_{L^{1}(J)} \\
& +\sum_{J \in \mathcal{J}}\left\|\sum_{P \in T:\left|I_{P}\right|>|J|} \epsilon_{P}\left\langle f, \phi_{1 P}\right\rangle \phi_{1 P} 1_{E_{2 P}}\right\|_{L^{1}(J)} .
\end{aligned}
$$

To estimate (12), we calculate for each $J \in \mathcal{J}$ and $P \in T$ :

$$
\left\|\left\langle f, \phi_{1 P}\right\rangle \phi_{1 P} 1_{E_{2 P}}\right\|_{L^{1}(J)} \leq C \varepsilon \mu\left|I_{P}\right|\left(1+\operatorname{dist}\left(I_{P}, J\right)\left|I_{P}\right|^{-1}\right)^{-\nu} .
$$

Here, we have set $\varepsilon:=\operatorname{energy}(T)$ and $\mu:=\operatorname{mass}(T)$. Fix an integer $k$ with $2^{k} \leq|J|$. Consider all $P \in T$ with $\left|I_{P}\right|=2^{k}$, then the intervals $I_{P}$ are pairwise disjoint, disjoint from $J$, and contained in $I_{T}$. Hence

$$
\sum_{P \in T:\left|I_{P}\right|=2^{k}}\left|I_{P}\right|\left(1+\operatorname{dist}\left(I_{P}, J\right)\left|I_{P}\right|^{-1}\right)^{-\nu} \leq C 2^{k}\left(1+\operatorname{dist}\left(I_{T}, J\right)\left|I_{T}\right|^{-1}\right)^{-\nu^{\prime}} .
$$

These estimates, summed over $2^{k} \leq|J|$ and $J \in \mathcal{J}$, yield no more than $C\left|I_{T}\right|$. Together with (14) this gives the desired bound for (12).

We consider (13). We can assume that the summation runs only over those $J \in \mathcal{J}$ for which there exists a $P \in T$ with $|J|<\left|I_{P}\right|$. Then we have $J \subset 3 I_{T}$ and $|J|<\left|I_{T}\right|$ for all $J$ occuring in the sum.

Fix an interval $J \in \mathcal{J}$ and observe that

$$
G_{J}:=J \cap \bigcup_{P \in T:\left|I_{P}\right|>|J|} E_{2 P}
$$

has measure at most $C \mu|J|$. Indeed, let $J^{\prime}$ be the dyadic interval which contains $J$ and $\left|J^{\prime}\right|=2|J| \leq\left|I_{T}\right|$. By maximality of $J, 3 J^{\prime}$ contains an interval $I_{P}$ for some $P \in T$. Let $P^{\prime}$ be the tile with $\left|I_{P^{\prime}}\right|=\left|J^{\prime}\right|$ and $P<P^{\prime}<I_{T} \times \omega_{T}$. Then $G_{J} \subset J \cap E_{P^{\prime}}$. And since $\operatorname{mass}(\{P\}) \leq \mu$, our claim follows.

Let $T_{2}$ be the 2 -tree of all $P \in T$ such that $\omega_{2 T} \subset \omega_{2 P}$, and let $T_{1}=T \backslash T_{2}$. Define, for $j=1,2$,

$$
F_{j J}:=\sum_{P \in T_{j}:\left|I_{P}\right|>|J|} \epsilon_{P}\left\langle f, \phi_{1 P}\right\rangle \phi_{1 P} 1_{E_{2 P}} .
$$

If $P, P^{\prime}$ are in the 1 -tree $T_{1}$ and have different scales, then the sets $\omega_{2 P}, \omega_{2 P^{\prime}}$ are disjoint and so are the sets $E_{2 P}, E_{2 P^{\prime}}$. Therefore, by considering single scales seperately one can estimate the $L^{\infty}$ norm of $F_{1 J}$ by $C \epsilon$. Hence

$$
\left\|F_{1 J}\right\|_{L^{1}(J)} \leq C \varepsilon\left|G_{J}\right| \leq C \varepsilon \mu|J| .
$$


Summing over the disjoint intervals $J \subset 3 I_{T}$ gives the desired estimate for the $T_{1}$-part of (13).

Fix $x$ and assume that $F_{2 J}(x)$ is not zero. Since the intervals $\omega_{2 P}$ with $P \in T_{2}$ are nested, there is a largest (smallest) interval $\omega_{+}\left(\omega_{-}\right)$of the form $\omega_{P}\left(\omega_{2 P}\right)$ with $P \in T_{2}, x \in E_{2 p}$ and $\left|I_{P}\right|>|J|$. In other words, we have for any $P \in T$ that $x \in E_{2 P}$ and $\left|I_{P}\right|>|J|$ if and only if $\left|\omega_{-}\right|<\left|\omega_{P}\right| \leq\left|\omega_{+}\right|$.

Hence we can write $F_{2 J}(x)$ as

$$
\begin{gathered}
\sum_{P \in T_{2}:\left|\omega_{-}\right|<\left|\omega_{P}\right| \leq\left|\omega_{+}\right|} \epsilon_{P}\left\langle f, \phi_{1 P}\right\rangle \phi_{1 P}(x)= \\
\sum_{P \in T_{2}} \epsilon_{P}\left\langle f, \phi_{1 P}\right\rangle\left(\phi_{1 P} *\left(M_{c\left(\omega_{+}\right)} D_{0.1\left|\omega_{+}\right|^{-1}}^{1} \phi-M_{c\left(\omega_{-}\right)} D_{0.09\left|\omega_{-}\right|^{-1}}^{1} \phi\right)\right)(x) .
\end{gathered}
$$

The last equality is easily seen from the geometry of the supports of the functions $\widehat{\phi}_{1 P}$. Therefore we can estimate $\left|F_{2 J}(x)\right|$ by

$$
C \sup _{J \subset I} \frac{1}{|I|} \int_{I}\left|\sum_{P \in T_{2}} \epsilon_{P}\left\langle f, \phi_{1 P}\right\rangle \phi_{1 P}(z)\right| d z,
$$

which is constant on $J$.

But $F_{2 J} 1_{J}$ is supported on the set $G_{J}$ of measure $\leq C \mu|J|$, hence

$$
\begin{aligned}
\sum_{J \in \mathcal{J}: J \subset 3 I_{T}}\left\|F_{2 J}\right\|_{L^{1}(J)} & \leq C \sum_{J \in \mathcal{J}: J \subset 3 I_{T}} \mu|J| \sup _{J \subset I} \frac{1}{|I|} \int_{I}\left|\sum_{P \in T_{2}} \epsilon_{P}\left\langle f, \phi_{1 P}\right\rangle \phi_{1 P}(z)\right| d z \\
& \leq C \mu\left\|M\left(\sum_{P \in T_{2}} \epsilon_{P}\left\langle f, \phi_{1 P}\right\rangle \phi_{1 P}\right)\right\|_{L^{1}\left(3 I_{T}\right)} \\
& \leq C \mu\left|I_{T}\right|^{\frac{1}{2}}\left\|\sum_{P \in T_{2}} \epsilon_{P}\left\langle f, \phi_{1 P}\right\rangle \phi_{1 P}\right\|_{2} .
\end{aligned}
$$

Here $M$ denotes the Hardy Littlewood maximal function and we have used the maximal theorem.

We observe that for different scales of $P, P^{\prime} \in T_{2}$ the intervals $\omega_{1 P}$ and $\omega_{1 P^{\prime}}$ are disjoint and therefore the functions $\phi_{1 P}$ and $\phi_{1 P^{\prime}}$ are orthogonal. Thus, following the arguments to estimate (10), we estimate the $L^{2}$ norm in the last displayed expression by

$$
C\left(\sum_{P \in T_{2}}\left|\left\langle f, \phi_{1 P}\right\rangle\right|^{2}\right)^{\frac{1}{2}} \leq C\left|I_{T}\right|^{\frac{1}{2}} \varepsilon
$$

This completes the desired estimate for the $T_{2}$-part of (13) and thereby finishes the proof of (8).

\section{References}

[1] L. Carleson, On convergence and growth of partial sums of Fourier series, Acta Math. 116 (1966), 135-157.

[2] C. Fefferman, Pointwise convergence of Fourier series, Ann. of Math. 98 (1973), 551-571.

[3] M.T. Lacey and C.M. Thiele, Bounds for the bilinear Hilbert transform on $L^{p}$, Proc. Nat. Acad. Sci. U.S.A. 94 (1997), 33-35. 
[4] _ On Calderòn's Conjecture, Ann. of Math. 149 (1999), 475-496.

[5] - On Calderòn's Conjecture for the bilinear Hilbert transform, Proc. Nat. Acad. Sci. 95 (1998), 4828-4830.

[6] $\ldots L^{p}$ Bounds for the bilinear Hilbert transform, $p>2$, Ann. of Math. 146 (1997), $693-724$.

School of Mathematics, Georgia Institute of Technology, Atlanta, GA 30332

E-mail address: lacey@math.gatech.edu

Department of Mathematics, UClA, Los Angeles CA 90095-1555

E-mail address: thiele@math.ucla.edu 